\section{OP88 LINK WORKING AT THE INTERSECTIONS: AN ETHNOGRAPHIC EXPLORATION OF DELIVERING SOCIAL PRESCRIBING IN PRIMARY CARE}

${ }^{1}$ Bethan Griffith*, 'Suzanne Moffatt, ${ }^{2}$ Tessa Pollard. 'Institute of Population Health Science, Newcastle University, Newcastle, UK; ${ }^{2}$ Department of Anthropology, Durham University, Durham, UK

\subsection{6/jech-2021-SSMabstracts.88}

Background Link worker social prescribing is increasingly accessed and delivered through UK NHS primary care, with plans to refer 900,000 patients to link workers by 2023 . However, learning is still needed about models of delivery and embedding of link workers. We present the results of an ethnography aimed at exploring how a social prescribing intervention in North-East England was becoming embedded locally from the perspectives of the link workers delivering it. The intervention, established in 2015 and funded via a Social Impact Bond (SIB), supported patients with Long Term Health Conditions (LTCs) using two local provider organisations.

Methods Ethnographic fieldwork was conducted between August 2019 and June 2020. Link workers consented for the ethnographer to undertake participant observation at their provider offices $(n=24)$. Link workers were then invited to participate in interviews $(n=5)$ and one of three focus groups $(n=17)$. The ethnographer also shadowed eight link workers. This included accompanying them seeing clients in GP surgeries, provider offices or in homes, and whilst they completed administrative tasks and attended staff meetings and training. Data included field notes and interview and focus group transcripts which were coded using thematic analysis in an iterative approach that engaged with complexity, context and existing knowledge.

Results Link workers operated in the liminal spaces between the intervention, primary care and the local community. These spaces were shaped by their own social location as well as that of their clients and the health and community workers they encountered. The subtle hegemony of primary care shaped experiences of working within these intersections, requiring link workers to negotiate a space and work hard securing referrals. Completion of the patient recorded outcome measures required for payment through the SIB also shaped work routines. The combined effect was to obstruct some routines, such as face to face meetings, whilst encouraging others, such as telephoning clients to secure referrals and complete PROMs. These routines contributed to a high staff turnover.

Conclusion As the NHS rolls out social prescribing it is crucial to consider different models of delivery and how to embed them. Shared understandings and strong relationships with primary care will be needed to ensure link workers receive referrals. Understanding that performative pressures to record specific outputs can shape the link worker role will also be important, not only in determining the nature of social prescribing being delivered, but in helping support link workers to stay in the role and become embedded in local teams.

\section{OP89 PREVALENCE AND INCIDENCE OF RECORDED NEUROMUSCULAR CONDITIONS IN PRIMARY CARE IN THE UK BETWEEN 2000 AND 2019}

${ }^{1}$ Iain Carey, ${ }^{1}$ Emma Banchoff, ${ }^{2}$ Niranjanan Nirmalananthan, ${ }^{1}$ Tess Harris, ${ }^{1}$ Stephen DeWilde, 'Umar Chaudhry*, 'Derek Cook. ${ }^{1}$ Population Health Research Institute, St George's, University of London, London, UK; ${ }^{2}$ Atkinson Morley Regional Neurosciences Centre, St George's University Hospitals NHS Foundation Trust, London, UK

\subsection{6/jech-2021-SSMabstracts.89}

Background Large-scale electronic primary care datasets can provide up-to-date, accurate epidemiological information on rarer diseases. In the UK, approximately 70,000 people are estimated to be living with neuromuscular disease (NMD), but this approximation is over 10 years old and used historical estimates from different sources. While GPs are unlikely to make NMD diagnoses themselves, specialist diagnoses from hospital discharges and clinic letters are well recorded and electronically searchable within primary care records. The recent expansion of the Clinical Practice Research Datalink (CPRD) allows timely reporting on the recorded incidence and prevalence of NMD in the UK over the last 20 years.

Methods A harmonised dataset of CPRD GOLD and Aurum was developed to provide annual denominators of actively registered patients between 2000 and 2019. A Read code hierarchy was developed for a wide range of NMDs; the database was then searched electronically to estimate incidence and (lifetime) prevalence rates in each year. To compare trends over time, rates were age standardised to the most recent CPRD population (2019).

Results The combined CPRD database contained approximately 13 million patients actively registered in each year. By 2019, 28,230 active patients had ever received a Read code for one of the NMDs (223.6 per 100,000), which would scale up to approximately 149,000 people in the UK. Lifetime prevalence rates per 100,000 were higher among males (239.0) than females (208.3). The most common classifications were Guillain-Barre syndrome (40.1), myasthenia gravis (33.7), muscular dystrophy (29.5), Charcot-Marie-Tooth (29.5) and inflammatory myopathies (25.0). Since 2000, overall prevalence grew by $63 \%$ but incidence remained steady over time. All age groups showed an increase in prevalence, with the largest increases seen at older ages ( $\geq 65$-years). Among individual conditions since about 2008, incidence of myasthenia gravis has been steadily rising while new cases of muscular dystrophy fell over the same period.

Conclusions Lifetime recording of many NMDs on primary care records exceeds current estimates of people living with these conditions; these are important data for health service and care planning. Temporal trends suggest this number is steadily increasing and there may be twice as many people living with these conditions than currently thought. While this may be partially due to increases in recording, it cannot be simply explained by new cases as incidence is largely unchanged over time. The increase in prevalence among older ages suggest increases in life expectancy among those living with NMDs may have taken place. 